\title{
A Reaction-Diffusion System for Leukemia Disease
}

\author{
Mohammed Benmir ${ }^{1 *}$, Soumaya Boujena ${ }^{1}$, Vitaly Volpert ${ }^{2}$ \\ ${ }^{1}$ MACS, Faculty of Sciences, University of Hassan II, 20100 Casablanca, Morocco. \\ 2 ICJ, UMR 5208 CNRS, University of Lyon 1, 69622 Villeurbanne, France. \\ * Corresponding author. Tel.: (+212)620021986; email: med.benmir@gmail.com \\ Manuscript submitted May 27, 2016; accepted November 30, 2016. \\ doi: 10.17706/ijapm.2017.7.2.87-92
}

\begin{abstract}
In this work, we propose a mathematical model based on reaction-diffusion equations to describe the development of normal and leukemic hematopoiesis. Specifically, we introduce a parameter that characterizes the strength of mutation. According to its value, leukemia will or will not develop. On the other hand, we show the existence of a traveling wave providing a transition from an equilibrium to other.
\end{abstract}

Key words: Leukemia, reaction-diffusion equations, monotone system.

\section{Introduction}

Erythropoiesis is a process of producing red blood cells (erythrocytes), which occurs primarily in the bone marrow. Erythropoiesis begins with engaged erythroid progenitors that differentiate into more mature cells, the erythroblasts, which differentiate into reticulocytes thereafter. In mammals, anucleated reticulocytes leave the bone marrow by entering the bloodstream where they become mature erythrocytes.

In adults, erythropoiesis occurs in the medullary cavities of flat bones [1].

Erythropoiesis is the process of production and regulation of red blood cells. It is step of a more general process, hematopoiesis that is a process in which three main cell types are produced and regulated: the red blood cells (or erythrocytes), the white blood cells (or leukocytes) and the platelets (or thrombocytes). It is the set of mechanisms that ensure the production and renewal of different blood cells. The Abnormalities in this developmental program lead to blood cell diseases including leukemia.

Hematopoiesis is a very intense and a very complex process with many levels of regulation: intracellular, extracellular, from other organs. These mechanisms control self-renewal, differentiation and apoptosis of hematopoietic cells and the response to various physiological situations, such as hypoxia, bleeding or infection. If some of these control mechanisms do not work, then various blood diseases including leukemia can develop.

Leukemia is type of cancer that affects the blood cells. It starts in the bone marrow, the soft tissue inside most bones, where blood cells are made. In this disease abnormal white blood cells are produced. Those cells, called leukemia cells, don't function as normal white blood cells because they grow faster than normal cells and they don't stop growing when they should to do it. There are several different types of leukemia. In general, each type of leukemia is carachterized by how fast it gets worse and the kind of white blood cells it affects.

The leukemia may be acute or chronic. Acute leukemia gets worse very fast and may make you feel sick right away. Chronic leukemia gets worse slowly and may not cause symptoms for years. Also the leukemia may be lymphocytic or myelogenous. Lymphocytic (or lymphoblastic) leukemia affects white blood cells 
called lymphocytes. Myelogenous leukemia affects the other type of cells that normally become granulocytes, red blood cells, or platelets.

It is a blood cancer characterized by an abnormal proliferation of immature blood cells, in the bone marrow.

From a mathematical point of view, the modeling of leukemia or any other pathology linked to the deregulation of the blood cell manufacturing mechanism is still in the embryonic stage. There is as yet no research project dedicated to the mathematical modeling of these phenomena. The best known overseas team interested in the modeling of hematopoiesis using partial differential equation systems [1] is directed by M.C. Mackey of McGill University in Canada. In the majority of cases, these are structured models in age and maturity (level of cell development). These approaches allow to take into account the different stages of development of the cells (primitive stem cells, progenitors, precursors and mature cells mainly).

We discuss here leukemia development. In particularly erythroleukemia is characterized by a rapid increase of erythroid progenitors in the bone marrow and the blood. A number of mathematical models has been proposed to understand hematopoiesis ([2]-[7]) and erythropoiesis ([8]-[10]). In [11] the authors investigate erythroid cell dynamics by means of a reaction-diffusion system describing cell dynamics and a system of ordinary differential equations describing concentrations of intra-cellular proteins involved in the regulation of self-renewal, differentiation and apoptosis. This model is used to focus on erythroleukemia. They modeled normal and leukemic erythropoiesis by reaction-diffusion systems, introducing the notion of strength of mutation. If this parameter exceeds a critical value, the leukemia will develop. In the case where the mutation is not strong enough, the leukemia will not develop.

We focus in this work on the development of normal and leukemic erythropoiesis modeled by reaction-diffusion systems, taking into account that the mutated cells can interact with each other.

In Section 2, we present the results of the existence and stability of leukemic equilibrium and we give the numerical examples and simulations in Section 3, and finally in section 4 we show the existence of traveling waves in the bistable case.

\section{Model}

Table 1. Table of Parameters

\begin{tabular}{|c|l|}
\hline Parameters & \multicolumn{1}{|c|}{ Meaning } \\
\hline$D_{P}$ & diffusion rate of normal cells \\
\hline$D_{Q}$ & diffusion rate of mutated cells \\
\hline$s$ & self-renewal rate of normal cells \\
\hline$d$ & differentiation rate of normal cells \\
\hline$a$ & apoptosis rate of normal cells \\
\hline$s_{m}$ & self-renewal rate of mutated cells \\
\hline$d_{m}$ & differentiation rate of mutated cells \\
\hline$a_{m}$ & apoptosis rate of mutated cells \\
\hline$H$ & constant flux of hematopoietic stem cells differentiating in erythroid progenitors \\
\hline$P_{0}$ & constant of maximal cell density \\
\hline
\end{tabular}

Consider two cell lineages, normal and mutated. The lineage of mutated cells will differ by the rates of self-renewal, differentiation and apoptosis. Mutated cells can self-renew more and differentiate less than normal cells. Consequently, the number of immature cells or blasts will increase replacing other cells, possibly resulting in the development of acute leukemia.

Denote by P cell from the normal lineage and by Q cell from the mutated lineage. We are interested in this 
work in the reaction-diffusion system of equations considered as a model of leukemia development:

$$
(S)\left\{\begin{array}{l}
\frac{\partial P}{\partial t}=D_{P} \frac{\partial^{2} P}{\partial x^{2}}+H+(s-d) P\left(P_{0}-P-Q\right)-a P \\
\frac{\partial Q}{\partial t}=D_{Q} \frac{\partial^{2} Q}{\partial x^{2}}+\left(s_{m}-d_{m}\right) Q^{2}\left(P_{0}-P-Q\right)-a_{m} Q,
\end{array}\right.
$$

where the parameters are presented in the following table:

The positive numbers $a, a_{m}, P_{0}, H, s, d, s_{m}$ and $d_{m}$ are such $s>d$ and $s_{m}>d_{m}$. In the following, we put $k=s-d, k_{m}=s_{m}-d_{m}$ and $\mu=\frac{a_{m} k}{k_{m} a}$ then we obtain,

$$
(S)\left\{\begin{array}{l}
\frac{\partial P}{\partial t}=D_{P} \frac{\partial^{2} P}{\partial x^{2}}+H+k P\left(P_{0}-P-Q\right)-a P \\
\frac{\partial Q}{\partial t}=D_{Q} \frac{\partial^{2} Q}{\partial x^{2}}+k_{m} Q^{2}\left(P_{0}-P-Q\right)-a_{m} Q
\end{array}\right.
$$

\section{Existence and Stability of Leukemic Equilibrium}

In this section, we study the existence and stability of equilibrium points of system (S).

\section{Proposition 1}

Existence and stability of the equilibrium points for the system (S), is given by the following two cases:

1) If $\frac{2 a}{k}<P_{0}, \frac{\left(\mu+\frac{a}{k}-\frac{H}{a}-P_{0}\right)^{2}}{3} \geq-\frac{H}{a}\left(\frac{2 a}{k}-P_{0}\right)$ and $\mu+\frac{a}{k}-\frac{H}{a}-P_{0}<0$ then the system admits three equilibrium points, and it is bistable when $\frac{1}{\mu}>P_{0}-\frac{a}{k}+\frac{H}{a}$,

2) If $\frac{2 a}{k} \geq P_{0}$ then the system (S) admits three equilibrium points, it is bistable when $\frac{1}{\mu}>\frac{a}{k}$.

\section{Remark 2}

The inverse parameter $\frac{1}{\mu}$ is called the strength of mutation. It characterizes the advantage of mutated cells over normal cells from the point of view of their survival and proliferation. Indeed, if the rate of self-renewal sm of mutated cells increases, then the value of $\frac{1}{\mu}$ also increases. If the rates of differentiation $\mathrm{dm}$ and of apoptosis am decrease, then, again, $\frac{1}{\mu}$ grows. Hence increase of this parameter corresponds to greater self-renewal and lesser differentiation and apoptosis of mutated cells in comparison with normal cells.

Consider a numerical simulation for the equilibrium of leukemia in the bistable case. 
Let $f(P)=P_{0}-P-\frac{a P-H}{k P}$ and $g(P)=\frac{a \mu P}{a P-H}$.

The following figure represents a bistable case under conditions of (1.1) in proposition for the leukemic state such that $H=1 ; k=1 ; k_{m}=1 ; P_{0}=5 ; a=1 ; a_{m}=1$.

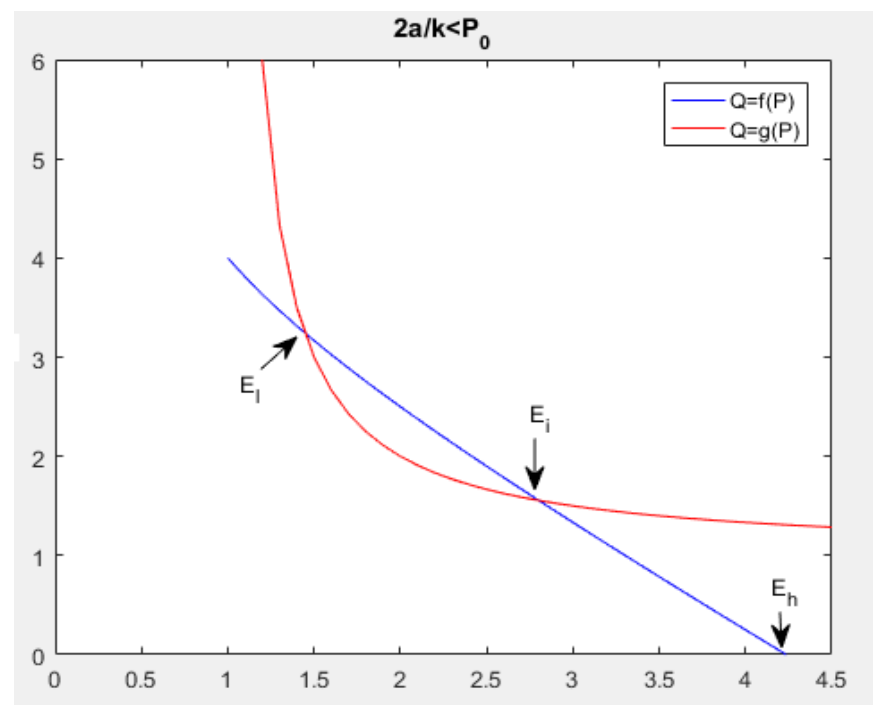

Fig. 1. Case (1.1) of proposition, the system (S) is stable around the equilibrium points $E_{h}, E_{l}$ and unstable around $E_{i}$.

\section{Existence of Travelling Waves in the Bistable Case}

The analysis of the ordinary differential system of equations allows us to do some conclusions about the behavior of solutions of a reaction-diffusion system (S). Precisely, classical results on monotone systems allow to conclude to the existence of a traveling wave providing a transition between healthy equilibrium and the leukemic equilibrium in the bistable case. The existence result is stated in the next theorem.

\section{Theorem 3}

In the bistable case, there exists a unique traveling wave solution connecting the healthy equilibrium $E_{h}$ and the leukemic equilibrium $E_{l}$, i.e. there exists a constant $c$ and $a$ vector valued function $Z(x, t)=W(x-c t)$, solution of system $(\mathrm{S})$ and such that $\lim _{x \rightarrow \infty} W(x)=W_{ \pm}$with $W_{-}=E_{l}$ and $W_{+}=E_{h}$.

\section{Proof.}

We put $P=U$ and $P_{0}-Q=V$, then the system (S) becomes:

$$
\left(S^{\prime}\right)\left\{\begin{array}{l}
\frac{\partial U}{\partial t}=D_{U} \frac{\partial^{2} U}{\partial x^{2}}+H+k U(V-U)-a U, \\
\frac{\partial V}{\partial t}=D_{V} \frac{\partial^{2} V}{\partial x^{2}}+k_{m}\left(P_{0}-V\right)^{2}(V-U)-a_{m}\left(V-P_{0}\right) .
\end{array}\right.
$$

Denoting $Z(U, V)=\left(\begin{array}{l}Z_{1} \\ Z_{2}\end{array}\right)=\left(\begin{array}{l}H+k U(V-U)-a U \\ k_{m}\left(P_{0}-V\right)^{2}(V-U)-a_{m}\left(V-P_{0}\right)\end{array}\right)$. 
We have $\frac{\partial Z_{1}}{\partial V}=k U>0$ and $\frac{\partial Z_{2}}{\partial U}=k_{m}\left(P_{0}-V\right)^{2}>0$.

Then the system $\left(\mathrm{S}^{\prime}\right)$ is monotone. The monotony of the system ensures the existence and uniqueness of traveling wave solutions in the bistable case (see [12],[13]).

\section{Acknowledgment}

This work has been supported with a grant PHC Volubilis from the French foreign office and the Moroccan ministry of education and research MA/11/246 and partially supported by LIRIMA.

\section{References}

[1] Blair, J., Mackey, M. C., \& Mahaffy. J. M. (1995). Age-structured and two delay models for erythropoiesis. Math. Biosci., 128, 317346.

[2] Colijn, C., \& Mackey, M. C. (2005). A mathematical model of hematopoiesis-I. Periodic chronic myelogenous leukemia. J. Theor. Biol., 237, 117132.

[3] Colijn, C., \& Mackey, M. C. (2005). A mathematical model of hematopoiesis-II. Cyclical neutrope- nia. J. Theor. Biol., 237, 133146.

[4] Adimy, M., Crauste, F., \& Abdllaoui, A. E. (2006). Asymptotic behavior of a discrete maturity structured system of hematopoietic stem cells dynamics with several delays. Math. Model. Nat. Phenom., 1(2), $1-22$.

[5] Adimy, M., et al. (2005). A mathematical study of the hematopoiesis process with applications to chronic myelogenous leukemia. SIAM J. Appl. Math., 65, 1328-1352.

[6] Adimy, M., et al. (2006). Modelling hematopoiesis mediated by growth factors with applications to periodic hematological diseases. Bull. Math. Biol., 68, 2321-2351.

[7] Bessonov, N., et al. (2006). Cell modelling of hematopoiesis. Math. Model. Nat. Phenom., 1(2), 81-103.

[8] Bessonov, N., et al. (2012). Mathematical modeling of erythropoiesis in vivo with multiple erythroblastic islands. Applied Mathematics Letters, 25, 1217-1221.

[9] Crauste, F., et al. (2008). Adding self-renewal in committed erythroid progenitors improves the biological relevance of a mathematical model of erythropoiesis. J. Theor. Biology, 250, 322-338.

[10] Demin, I., et al. (2010). A multi-scale model of erythropoiesis. Journal of Biological Dynamics, 4, 59-70.

[11] Bessonov, N., et al. (2009). Dynamics of erythroid progenitors anderythroleukemia. Math. Model. Nat. Phenom., 4(3), 210-232.

[12] Volpert, A., et al. (1994). Travelling wave solutions of parabolic systems. AMS, Providence.

[13] Volpert. V. (2014). Elliptic partial differential equations. Reaction-Diffusion Equations. Birkhäuser.

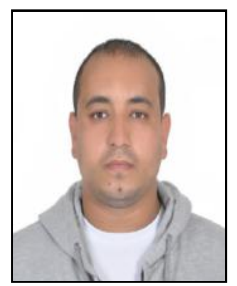

Mohammed Benmir was born in Tangier Morocco, in 2009. He received the licence degree in mathematics and applications and the master degree in modeling and systems from Faculty of Sciences, University of Hassan II, Casablanca, Morocco, in 2009, 2012 respectively. He is currently pursuing his Ph.D. degree in reaction-diffusion waves in cell population dynamics in the same faculty. He participated in the works that was published as 'V.Volpert, N.Reinberg, M.Benmir, S.Boujena. Existence of pulses for a monotone system. Accepted to Journal of Nonlinear Analysis', 'M.Benmir,N.Bessonov,S.Boujena,V.Volpert. Travelling Waves of Cell Differentiation. Accepted to Journal of Acta Biotheoretica'. And conference proceeding 'M.Benmir,N.Bessonov,S.Boujena,V.Volpert. Multi-scale hybrid model of cell differentiation propagation as 
traveling waves. In Proceedings of NumAn2014, 6th Conference on Numerical Analysis'. His research fields are the mathematics applied in biology.

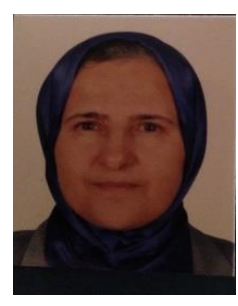

S. Boujena was born in Casablanca, Morocco in May 1959. She is a Professor-researcher at Sciences Faculty Ain Chock in Casablanca, Morocco and member of MACS and LIRIMA Laboratories. She obtained the DEA degree in 1984 and the PHD in 1987 in Numerical Analysis at the University Pierre and Marie Curie in Paris, France. She obtained the post-doctoral thesis in 2002 at Mohammadia School of Engineering of Rabat, Morocco. She is teaching in Sciences Faculty Ain Chock since September 1987 and here works are about modeling, mathematical and numerical analysis of image processing, transport of nutrients in growing plants, fluid flow, interaction fluid-structure, and cancer modeling.

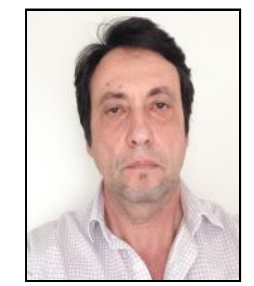

Vitaly Volpert graduated and obtained PhD in Physical and Mathematical Sciences at the Institute of Physical Chemistry of the Academy of Sciences of Russia. He is now a director of Research, CNRS UMR 5208, University of Lyon 1, France. Prof Volpert is an organizer and chief editor of the Mathematical modelling of natural phenomena and the European Communications, edition of the European Society of Mathematical and Theoretical Biology. 